\title{
Editorial
}

\section{Acknowledgement to Reviewers of Societies in 2013}

Societies Editorial Office, MDPI AG, Klybeckstrasse 64, CH-4057 Basel, Switzerland

Published: 26 February 2014

The editors of Societies would like to express their sincere gratitude to the following reviewers for assessing manuscripts in 2013:

$\begin{array}{lll}\text { Abbinnett, Ross } & \text { Greitemeyer, Tobias } & \text { Lin, Tammy } \\ \text { Andersson, Thomas } & \text { Gullberg, Mats T. } & \text { Lye, Jenny } \\ \text { Armstrong, Justin } & \text { Hand, Martin } & \text { Mack, Michael } \\ \text { Asano, Motoshi } & \text { Hand, Richard } & \text { Marder, Michael } \\ \text { Ashkenazi, Michael } & \text { Happ, Christian } & \text { Maya Jariego, Isidro } \\ \text { Bell, David } & \text { Hartoonian, Gevork } & \text { McCurley, Kay } \\ \text { Blümke, Matthias } & \text { Head, Lesley } & \text { McGarty, Craig } \\ \text { Borgatti, Stephen P. } & \text { Hicks, Diana } & \text { McKeown, Shelley } \\ \text { Borislavov, Rad } & \text { Hosek, Jennifer Ruth } & \text { Melzer, André } \\ \text { Bouman, Thijs } & \text { Houle, Karen } & \text { menninghaus, winfried } \\ \text { Braester, Yomi } & \text { Howell, Sally } & \text { Michelson, William } \\ \text { Brockmyer, Jeanne } & \text { Hummer, Tom A. } & \text { Moosavi, Leon } \\ \text { Burke, Deirdre } & \text { Instone, Lesley } & \text { Murphy, Eleanor } \\ \text { Calvo, Paco } & \text { Jones, Michael } & \text { Narine, Neil } \\ \text { Collie, Natalie } & \text { Jones, Nora L. } & \text { Noland, Marcus } \\ \text { Cross, Ian } & \text { Jones, Stephen } & \text { O’Toole, Therese } \\ \text { Cummins, Robert A. } & \text { Keeble, Richard } & \text { Procter, Nicholas G. } \\ \text { Duong, Mylien T. } & \text { Krase, Jerome } & \text { Pyżalski, Jacek } \\ \text { Ehland, Christoph } & \text { Kueffer, Christoph } & \text { Radford, Lorraine } \\ \text { Ferguson, Christopher J. } & \text { Kuhn, Annette } & \text { Ramirez, Marizen } \\ \text { Forrest, Tara } & \text { Kumar, Anup } & \text { Ray, James } \\ \text { Freeman, Lindsey } & \text { Kusters, Annelies } & \text { Richter, Jörg } \\ \text { Gaspar, Tania } & \text { Lawrence, Christopher M. } & \text { Riggs, Damien } \\ \text { Gavin, Alice } & \text { Lazzaro, Elisabetta } & \text { Rodriguez, V. } \\ \text { Gentile, Doug } & \text { Lee, Shawna J. } & \text { Ryan, Louise } \\ \text { Gorelick, Root } & \text { Leslie, Esther } & \text { Shapiro, Lauren Spies } \\ \text { Granter, Edward } & \text { Leveau, Arnaud } & \text { Shelton, Allen } \\ & & \end{array}$


Stueck, William

Tauber, Susanne

Taylor, Leslie K.

Tejeda Martín, Isabel

Tomescu, Alexandru M. F.
Tynkkynen, Liina-Kaisa

Vasquez, Eduardo

Vogelgesang, Jens

Wainwright, Emma

Warburton, Wayne
Watkins, Holly

Winter, Jay

Yolles, Maurice

(C) 2014 by the authors; licensee MDPI, Basel, Switzerland. This article is an open access article distributed under the terms and conditions of the Creative Commons Attribution license (http://creativecommons.org/licenses/by/3.0/). 